\title{
Article
}

\section{On generalization of extended Gegenbauer polynomials of two variables}

\author{
Ahmed Ali Al-Gonah ${ }^{1, *}$ and Ahmed Ali Atash ${ }^{2}$ \\ 1 Department of Mathematics, Faculty of Science, Aden University, Aden, Yemen. \\ 2 Department of Mathematics, Faculty of Education Shabwah, Aden University, Aden, Yemen. \\ * Correspondence: gonah1977@yahoo.com
}

Academic Editor: M. Mursaleen

Received: 21 January 2021; Accepted: 8 May 2021; Published: 11 June 2021.

\begin{abstract}
Recently, many extensions of some special functions are defined by using the extended Beta function. In this paper, we introduce a new generalization of extended Gegenbauer polynomials of two variables by using the extended Gamma function. Some properties of these generalized polynomials such as integral representation, recurrence relation and generating functions are obtained.
\end{abstract}

Keywords: Gamma function; Extended Gegenbauer polynomials; Integral representations; Generating functions.

MSC: 33B15; 33B20; 33C05; 33C15; 33C45; 33C47; 33C50.

\section{Introduction}

I

$\mathrm{n}$ recent years, several extensions of the well known special functions have been considered by several authors [1-5]. Extensions of Euler's Gamma function together with the set of related higher transcendental special functions were introduced by Chaudhry and Zubair [1] as:

$$
\Gamma_{p}(x)=\int_{0}^{\infty} t^{x-1} \exp \left(-t-p t^{-1}\right) d t, \quad \operatorname{Re}(p)>0, p=0, \operatorname{Re}(x)>0 .
$$

Clearly, we have $\Gamma_{0}(x)=\Gamma(x)$ where $\Gamma(x)$ is the well known classical Gamma function defined by [6]:

$$
\Gamma(x)=\int_{0}^{\infty} t^{x-1} e^{-t} d t, \quad \operatorname{Re}(x)>0 .
$$

The integral (1) can be presented as (see [1, p.101] for $x=0$ ) also [2, p.79]:

$$
a^{-x} \Gamma_{a p}(x)=\int_{0}^{\infty} t^{x-1} \exp \left(-a t-p t^{-1}\right) d t,
$$

where $a+p>0, \operatorname{Re}(a)>0, p=0, \operatorname{Re}(x)>0$, which for $a=1$ reduces to (1).

Here in this paper, we denote the right hand side of (3) as $\Gamma_{(a, p)}(x)$, i.e.,

$$
\Gamma_{(a, p)}(x)=\int_{0}^{\infty} t^{x-1} \exp \left(-a t-p t^{-1}\right) d t
$$

where $a+p>0, \operatorname{Re}(a)>0, p=0, \operatorname{Re}(x)>0$.

Note that,

$$
\begin{aligned}
& \Gamma_{(a, p)}(x)=a^{-x} \Gamma_{a p}(x), \\
& \Gamma_{(1, p)}(x)=\Gamma_{p}(x), \\
& \Gamma_{(a, 0)}(x)=a^{-x} \Gamma(x),
\end{aligned}
$$




$$
\Gamma_{(1,0)}(x)=\Gamma(x) .
$$

Recently, Dattoli et al., [7] introduced the extended Gegenbauer polynomials of two variable $C_{n}^{\alpha}(x, y ; a)$ as follows:

$$
C_{n}^{\alpha}(x, y ; a)=\frac{1}{\Gamma(\alpha)} \sum_{k=0}^{\left[\frac{n}{2}\right]} \frac{(2 x)^{n-2 k}(-y)^{k} \Gamma(\alpha+n-k)}{k !(n-2 k) ! a^{\alpha+n-k}},
$$

which specified by the following generating function and integral representation:

$$
\begin{aligned}
& \sum_{n=0}^{\infty} C_{n}^{\alpha}(x, y ; a) t^{n}=\left(a-2 x u+y u^{2}\right)^{-\alpha}, \\
& C_{n}^{\alpha}(x, y ; a)=\frac{1}{\Gamma(\alpha) n !} \int_{0}^{\infty} t^{\alpha-1} \exp (-a t) H_{n}(2 x t,-y t) d t,
\end{aligned}
$$

where $H_{n}(x, y)$ denotes the 2-variable Hermite-Kampé de Fériet polynomials defined by [8]:

$$
H_{n}(x, y)=n ! \sum_{k=0}^{\left[\frac{n}{2}\right]} \frac{y^{k} x^{n-2 k}}{k !(n-2 k) !}
$$

and specified by the following generating function:

$$
\exp \left(x t+y t^{2}\right)=\sum_{n=0}^{\infty} H_{n}(x, y) \frac{t^{n}}{n !} .
$$

In many recent works (see for example [9-12]), the extended Beta function and its systemic generalizations are used to introduce new extended special functions such as hypergeometric function, Appell's and Lauricella's hypergeometric functions, Mittag Leffler function and Zeta function. Very recently, in terms of the extended Gamma function defined in (1) Atash and Al-Gonah [13] introduced the extended Gegenbauer polynomials of two variables $C_{n}^{\alpha}(x, y ; p)$ as follows:

$$
C_{n}^{\alpha}(x, y ; p)=\frac{1}{\Gamma(\alpha)} \sum_{k=0}^{\left[\frac{n}{2}\right]} \frac{(2 x)^{n-2 k}(-y)^{k} \Gamma_{p}(\alpha+n-k)}{k !(n-2 k) !}
$$

where $\operatorname{Re}(p)>0, \quad p=0, \operatorname{Re}(\alpha+n-k)>0$, which specified by the following generating function and integral representation:

$$
\begin{aligned}
& \sum_{n=0}^{\infty} C_{n}^{\alpha}(x, y ; p) u^{n}=\frac{\left(1-2 x u+y u^{2}\right)^{-\alpha}}{\Gamma(\alpha)} \Gamma_{\left(1-2 x u+y u^{2}\right) p}(\alpha) \\
& C_{n}^{\alpha}(x, y ; p)=\frac{1}{\Gamma(\alpha) n !} \int_{0}^{\infty} t^{\alpha-1} \exp \left(-t-p t^{-1}\right) H_{n}(2 x t,-y t) d t
\end{aligned}
$$

From Equations (9) and (14), we have

$$
\begin{aligned}
& C_{n}^{\alpha}(x, y ; a)=\frac{1}{a^{\alpha}} C_{n}^{\alpha}\left(\frac{x}{a}, \frac{y}{a} ; 0\right), \\
& C_{n}^{\alpha}(x, y ; a=1)=C_{n}^{\alpha}(x, y ; p=0)=C_{n}^{\alpha}(x, y)=\frac{1}{\Gamma(\alpha)} \sum_{k=0}^{\left[\frac{n}{2}\right]} \frac{(2 x)^{n-2 k}(-y)^{k} \Gamma(\alpha+n-k)}{k !(n-2 k) !}, \\
& C_{n}^{\alpha}(x, y=1 ; a=1)=C_{n}^{\alpha}(x, y=1 ; p=0)=C_{n}^{\alpha}(x)=\frac{1}{\Gamma(\alpha)} \sum_{k=0}^{\left[\frac{n}{2}\right]} \frac{(2 x)^{n-2 k}(-1)^{k} \Gamma(\alpha+n-k)}{k !(n-2 k) !},
\end{aligned}
$$

where $C_{n}^{\alpha}(x)$ is the classical Gegenbauer polynomials [6]. 
Further, from Equations (9) and (14), we have

$$
\begin{aligned}
& C_{n}^{\alpha}(x, 1 ; a)=C_{n}^{\alpha}(x ; a)=\frac{1}{\Gamma(\alpha)} \sum_{k=0}^{\left[\frac{n}{2}\right]} \frac{(2 x)^{n-2 k}(-1)^{k} \Gamma(\alpha+n-k)}{k !(n-2 k) ! a^{\alpha+n-k}} \\
& C_{n}^{\alpha}(x, 1 ; p)=C_{n}^{\alpha}(x ; p)=\frac{1}{\Gamma(\alpha)} \sum_{k=0}^{\left[\frac{n}{2}\right]} \frac{(2 x)^{n-2 k}(-1)^{k} \Gamma_{(p}(\alpha+n-k)}{k !(n-2 k) !}
\end{aligned}
$$

where $C_{n}^{\alpha}(x ; p)$ is the extended Gegenbauer polynomials given in [13].

This paper is a further attempt in this direction to stress the importance of the use of extended Gamma function in introducing new extended special polynomials. The main object of this paper is to introduce a new generalization for the extended Gegenbauer polynomials defined in Equation (14) by using the extended Gamma function defined in Equation (4).

\section{A generalization of extended Gegenbauer polynomials}

In terms of the extended Gamma function $\Gamma_{(a, p)}(x)$ defined in (4), we introduce a new generalization of extended Gegenbauer polynomials of two variables, denoted by $C_{n}^{\alpha}(x, y ; a, p)$, as follows:

$$
C_{n}^{\alpha}(x, y ; a, p)=\frac{1}{\Gamma(\alpha)} \sum_{k=0}^{\left[\frac{n}{2}\right]} \frac{(2 x)^{n-2 k}(-y)^{k} \Gamma_{(a, p)}(\alpha+n-k)}{k !(n-2 k) !},
$$

where $a+p>0, \operatorname{Re}(p)>0, p=0, \operatorname{Re}(\alpha+n-k)>0$.

Remark 1. From Equation (22), we note that:

1. For $y=1$, Equation (22) reduces to the following new extended Gegenbauer polynomials $C_{n}^{\alpha}(x ; a, p)$ :

$$
C_{n}^{\alpha}(x ; a, p)=\frac{1}{\Gamma(\alpha)} \sum_{k=0}^{\left[\frac{n}{2}\right]} \frac{(2 x)^{n-2 k}(-1)^{k} \Gamma_{(a, p)}(\alpha+n-k)}{k !(n-2 k) !} .
$$

2. For $p=0$ and using relation (5), Equation (22) reduces to the extended Gegenbauer polynomials of two variables $C_{n}^{\alpha}(x, y ; a)$ defined in (9).

3. For $a=1$ and using relation (6), Equation (22) reduces to the extended Gegenbauer polynomials of two variables $C_{n}^{\alpha}(x, y ; p)$ defined in (14).

4. For $y=a=1$ and using relation (6), Equation (22) reduces to the extended Gegenbauer polynomials $C_{n}^{\alpha}(x ; p)$ defined in $(20)$.

Also, note that from Equations (22), (14), (23) and using relation (5), we have the following relations:

$$
\begin{aligned}
& C_{n}^{\alpha}(x, y ; a, p)=a^{-\alpha} C_{n}^{\alpha}\left(\frac{x}{a}, \frac{y}{a} ; a p\right), \\
& C_{n}^{\alpha}(x ; a, p)=a^{-\alpha} C_{n}^{\alpha}\left(\frac{x}{a}, \frac{1}{a} ; a p\right) .
\end{aligned}
$$

Now, we establish some properties for the generalization of extended Gegenbauer polynomials of two variables $C_{n}^{\alpha}(x, y ; a, p)$ in the form of the following theorems:

Theorem 1. The following integral representation for the new extended Gegenbauer polynomials $C_{n}^{\alpha}(x, y ; a, p)$ holds true:

$$
C_{n}^{\alpha}(x, y ; a, p)=\frac{1}{\Gamma(\alpha) n !} \int_{0}^{\infty} t^{\alpha-1} \exp \left(-a t-p t^{-1}\right) H_{n}(2 x t,-t y) d t
$$

Proof. Using Equations (22) and (17) in the left hand side of Equation (26), we get

$$
C_{n}^{\alpha}(x, y ; a, p)=\frac{1}{\Gamma(\alpha)} \int_{0}^{\infty} t^{\alpha-1} \exp \left(-a t-p t^{-1}\right) \sum_{k=0}^{\left[\frac{n}{2}\right]} \frac{(-t y)^{k}(2 x t)^{n-2 k}}{k !(n-2 k) !} d t
$$


Making use of Equation (12) in the right hand side of Equation (27), we get assertion (26) of Theorem 1.

For $y=1$ in assertion (26) of Theorem 1, we get the following result:

Corollary 1. The following integral representation for the new extended Gegenbauer polynomials $C_{n}^{\alpha}(x ; a, p)$ holds true:

$$
C_{n}^{\alpha}(x ; a, p)=\frac{1}{\Gamma(\alpha) n !} \int_{0}^{\infty} t^{\alpha-1} \exp \left(-a t-p t^{-1}\right) H_{n}(2 x t,-t) d t .
$$

Remark 2. From results (26) and (28), we note that:

1. For $p=0$, result (26) reduces to a known result (21) given in [7].

2. For $a=1$, result (26) reduces to a known result (16) given in [13].

3. For $a=1$, result (28) reduces to a known result given in [13].

Further, by making use of result (26), we get the following results for $C_{n}^{\alpha}(x, y ; a, p)$ :

Theorem 2. The following recurrence relation for the new extended Gegenbauer polynomials $C_{n}^{\alpha}(x, y ; a, p)$ holds true:

$$
(n+1) C_{n+1}^{\alpha}(x, y ; a, p)=2 \alpha x C_{n}^{\alpha+1}(x, y ; a, p)-2 \alpha y C_{n-1}^{\alpha+1}(x, y ; a, p)
$$

Proof. Consider the following recurrence relation [14]:

$$
H_{n+1}(x, y)=x H_{n}(x, y)+2 n y H_{n-1}(x, y)
$$

Replacing $x$ by $2 x t$ and $y$ by $-y t$ in relation (30) and then multiplying both sides by $\frac{t^{\alpha-1} \exp \left(-a t-p t^{-1}\right)}{\Gamma(\alpha) n !}$ and integrating the resultant equation with respect to $t$ between the limits 0 to $\infty$, we get

$$
\begin{aligned}
& \frac{1}{\Gamma(\alpha) n !} \int_{0}^{\infty} t^{\alpha-1} \exp \left(-a t-p t^{-1}\right) H_{n+1}(2 x t,-y t) d t \\
& =\frac{2 x}{\Gamma(\alpha) n !} \int_{0}^{\infty} t^{\alpha} \exp \left(-a t-p t^{-1}\right) H_{n}(2 x t,-y t) d t-\frac{2 y}{\Gamma(\alpha)(n-1) !} \int_{0}^{\infty} t^{\alpha} \exp \left(-a t-p t^{-1}\right) H_{n-1}(2 x t,-y t) d t
\end{aligned}
$$

which on using relation (26) yields assertion (29) of Theorem 2.

For $y=1$ in assertion (26) of Theorem 2, we get the following result:

Corollary 2. The following recurrence relation for the new extended Gegenbauer polynomials $C_{n}^{\alpha}(x ; a, p)$ holds true:

$$
(n+1) C_{n+1}^{\alpha}(x ; a, p)=2 \alpha x C_{n}^{\alpha+1}(x ; a, p)-2 \alpha C_{n-1}^{\alpha+1}(x ; a, p) .
$$

Remark 3. 1. Setting $p=0$ in result (30), we obtain a known result given in [7].

2. Setting $a=1$ in results (30) and (32), we obtain a known results given in [13].

Theorem 3. The following differential equation of the new extended Gegenbauer polynomials $C_{n}^{\alpha}(x, y ; a, p)$ holds true:

$$
\left(y \frac{\partial^{3}}{\partial x^{2} \partial p}+2 x \frac{\partial}{\partial x}-2 n\right) C_{n}^{\alpha}(x, y ; a, p)=0
$$

Proof. Consider the following differential equation [14]:

$$
\left(2 y \frac{\partial^{2}}{d x^{2}}+x \frac{\partial}{\partial x}-n\right) H_{n}(x, y)=0
$$

Replacing $x$ by $2 x t$ and $y$ by $-y t$ in relation (34) and using the relation

$$
\frac{\partial}{\partial(t x)}=\frac{1}{t} \frac{\partial}{\partial x}
$$


and then multiplying both sides by $\frac{t^{\alpha-1} \exp \left(-a t-p t^{-1}\right)}{\Gamma(\alpha) n !}$ and integrating the resultant equation with respect to $t$ between the limits 0 to $\infty$, we get

$$
\begin{aligned}
& -y \frac{\partial^{2}}{\partial x^{2}} \frac{1}{\Gamma(\alpha) n !} \int_{0}^{\infty} t^{\alpha-2} \exp \left(-a t-p t^{-1}\right) H_{n}(2 x t,-y t) d t \\
& +\left(2 x \frac{\partial}{\partial x}-2 n\right) \frac{1}{\Gamma(\alpha) n !} \int_{0}^{\infty} t^{\alpha-1} \exp \left(-a t-p t^{-1}\right) H_{n}(2 x t,-y t) d t=0 .
\end{aligned}
$$

Using relation (26) in the above equation and then using the following relation:

$$
\frac{\partial}{\partial p} C_{n}^{\alpha}\left(x, y_{i}, a, p\right)=(1-\alpha)^{-1} C_{n}^{\alpha-1}\left(x, y_{i}, a, p\right),
$$

in the first term of the resultant equation, we get the desired result.

For $y=1$ in assertion (33) of Theorem 3, we get the following result:

Corollary 3. The following differential equation of the new extended Gegenbauer polynomials $C_{n}^{\alpha}(x ; a, p)$ holds true:

$$
\left(\frac{\partial^{3}}{\partial x^{2} \partial p}+2 x \frac{\partial}{\partial x}-2 n\right) C_{n}^{\alpha}(x ; a, p)=0 .
$$

\section{Generating functions and other properties of $C_{n}^{\alpha}(x, y ; a, p)$}

Very recently, many generating functions for Gegenbauer polynomials and its extension are obtained (see for example $[13,15,16])$. Here we prove some generating functions for the new extended Gegenbauer polynomials $C_{n}^{\alpha}(x, y ; a, p)$ in the form of the following theorems:

Theorem 4. The following generating function for the new extended Gegenbauer polynomials $C_{n}^{\alpha}(x, y ; a, p)$ holds true:

$$
\sum_{n=0}^{\infty} C_{n}^{\alpha}(x, y ; a, p) u^{n}=\frac{1}{\Gamma(\alpha)} \Gamma_{\left(a-2 x u+y u^{2}, p\right)}(\alpha),
$$

where $\operatorname{Re}\left(a-2 x u+y u^{2}\right)>0$.

Proof. Using Equations (22) in the left hand side of Equation (39) and then putting $n=n+2 k$ in the resultant equation, we get

$$
\sum_{n=0}^{\infty} C_{n}^{\alpha}(x, y ; a, p) u^{n}=\frac{1}{\Gamma(\alpha)} \sum_{n=0}^{\infty} \frac{(2 x u)^{n}}{n !} \sum_{k=0}^{\infty} \frac{(-y)^{k} \Gamma_{(a, p)}(\alpha+n+k) u^{2 k}}{k !} .
$$

Now, using Equation (17) in the right hand side of the above equation, we obtain

$$
\sum_{n=0}^{\infty} C_{n}^{\alpha}(x, y ; a, p) u^{n}=\frac{1}{\Gamma(\alpha)} \int_{0}^{\infty} t^{\alpha-1} \exp \left(-t\left(a-2 x u+y u^{2}\right)-p t^{-1}\right) d t
$$

which in view of Equation (17) yields assertion (39) of Theorem 4.

For $y=1$ in assertion (39) of Theorem 4, we get the following result:

Corollary 4. The following generating function for the new extended Gegenbauer polynomials $C_{n}^{\alpha}(x ; a, p)$ holds true:

$$
\sum_{n=0}^{\infty} C_{n}^{\alpha}(x ; a, p) u^{n}=\frac{1}{\Gamma(\alpha)} \Gamma_{\left(a-2 x u+u^{2}, p\right)}(\alpha)
$$

where $\operatorname{Re}\left(a-2 x u+u^{2}\right)>0$. 
Remark 4. 1. Setting $p=0$ in result (39) and using relation (7), we obtain a known result (20) given in [7].

2. Setting $a=1$ in result (39) and then using relation (5), we obtain a known result (15) given in [13].

3. Setting $a=1$ in result (42) and then using relation (5), we obtain a known result given in [13].

Theorem 5. The following generating function for the new extended Gegenbauer polynomials $C_{n}^{\alpha}(x, y ; a, p)$ holds true:

$$
\sum_{n=0}^{\infty}(1+k)_{n} C_{n+k}^{\alpha}(x, y ; a, p) \frac{u^{n}}{n !}=C_{k}^{\alpha}\left(x-y u, y ; a-2 x u+y u^{2}, p\right) .
$$

Proof. Consider the following generating function [17, p.452]:

$$
\sum_{n=0}^{\infty} H_{n+k}(x, y) \frac{u^{n}}{n !}=\exp \left(x u+y u^{2}\right) H_{k}(x+2 y u, y) .
$$

Replacing $x$ by $x t$ and $y$ by $-y t$ in above equation and multiplying both sides by $\frac{t^{\alpha-1} \exp \left(-a t-p t^{-1}\right)}{\Gamma(\alpha) k !}$ and integrating the resultant equation with respect to $t$ from 0 to $\infty$, we get

$$
\begin{aligned}
& \sum_{n=0}^{\infty} \frac{(n+k) !}{k ! \Gamma(\alpha)(n+k) !} \int_{0}^{\infty} t^{\alpha-1} \exp \left(-a t-p t^{-1}\right) H_{n+k}(2 x t,-y t) \frac{u^{n}}{n !} d t \\
& =\frac{1}{k ! \Gamma(\alpha)} \int_{0}^{\infty} t^{\alpha-1} \exp \left[-\left(a-2 x u+y u^{2}\right) t-p t^{-1}\right] H_{k}(2(x-y u) t,-y t) d t
\end{aligned}
$$

which on using relation (26) yields assertion (43) of Theorem 5.

For $y=1$ in assertion (43) of Theorem 5, we get the following result:

Corollary 5. The following generating function for the new extended Gegenbauer polynomials $C_{n}^{\alpha}(x ; a, p)$ holds true:

$$
\sum_{n=0}^{\infty}(1+k)_{n} C_{n+k}^{\alpha}(x ; a, p) \frac{u^{n}}{n !}=C_{k}^{\alpha}\left(x-u ; a-2 x u+u^{2}, p\right) .
$$

Remark 5. 1. Setting $p=0$ in result (43) and using relation (7), we obtain a known result given in [7].

2. Setting $a=1$ in results (43) and (46), we have

$$
\begin{aligned}
& \sum_{n=0}^{\infty}(1+k){ }_{n} C_{n+k}^{\alpha}(x, y ; p) \frac{u^{n}}{n !}=C_{k}^{\alpha}\left(x-y u, y ; 1-2 x u+y u^{2}, p\right), \\
& \sum_{n=0}^{\infty}(1+k){ }_{n} C_{n+k}^{\alpha}(x ; p) \frac{u^{n}}{n !}=C_{k}^{\alpha}\left(x-u ; 1-2 x u+u^{2}, p\right),
\end{aligned}
$$

which in view of relations (24) and (25) gives a known results given in [13].

Theorem 6. The following generating function for the new extended Gegenbauer polynomials $C_{n}^{\alpha}(x, y ; a, p)$ holds true:

$$
\sum_{k=0}^{\infty} \sum_{n=0}^{\infty}(n+k) ! C_{n+k}^{\alpha}(x, y ; a, p) \frac{u^{n}}{n !} \frac{v^{k}}{k !}=\frac{1}{\Gamma(\alpha)} \Gamma_{(\Delta, p)}(\alpha),
$$

where $\Delta=a-2 x u+y u^{2}-2 x v+2 y u v+y v^{2}$.

Proof. Using relation (26) in the left hand side of Equation (49) and interchanging the order of the summation and integration, we get

$$
\sum_{k=0}^{\infty} \sum_{n=0}^{\infty}(n+k) ! C_{n+k}^{\alpha}(x, y ; a, p) \frac{u^{n}}{n !} \frac{v^{k}}{k !}=\frac{1}{\Gamma(\alpha)} \int_{0}^{\infty} t^{\alpha-1} \exp \left(-a t-p t^{-1}\right) \sum_{k=0}^{\infty} \sum_{n=0}^{\infty} H_{n+k}(2 x t,-y t) \frac{u^{n}}{n !} \frac{v^{k}}{k !} d t
$$


Now, using relation (44) in the right hand side of the above equation, we get

$$
\begin{aligned}
& \sum_{k=0}^{\infty} \sum_{n=0}^{\infty}(n+k) ! C_{n+k}^{\alpha}(x, y ; a, p) \frac{u^{n}}{n !} \frac{v^{k}}{k !} \\
& =\frac{1}{\Gamma(\alpha)} \int_{0}^{\infty} t^{\alpha-1} \exp \left(-a t+2 x t u-y t u^{2}-p t^{-1}\right) \sum_{k=0}^{\infty} H_{k}(2 x t-2 y t u,-y t) \frac{v^{k}}{k !} d t
\end{aligned}
$$

which on using relation (13) gives

$$
\sum_{k=0}^{\infty} \sum_{n=0}^{\infty}(n+k) ! C_{n+k}^{\alpha}(x ; a, p) \frac{u^{n}}{n !} \frac{v^{k}}{k !}=\frac{1}{\Gamma(\alpha)} \int_{0}^{\infty} t^{\alpha-1} \exp \left[-\left(a-2 x u+y u^{2}-2 x v+2 y u v+y v^{2}\right) t-p t^{-1}\right] d t
$$

Making use of relation (17) in the right hand side of Equation (52), we get assertion (49) of Theorem 6.

For $y=1$ in assertion (49) of Theorem 6 , we get the following result:

Corollary 6. The following generating function for the new extended Gegenbauer polynomials $C_{n}^{\alpha}(x ; a, p)$ holds true:

$$
\sum_{k=0}^{\infty} \sum_{n=0}^{\infty}(n+k) ! C_{n+k}^{\alpha}(x ; a, p) \frac{u^{n}}{n !} \frac{v^{k}}{k !}=\frac{1}{\Gamma(\alpha)} \Gamma_{(\Delta, p)}(\alpha)
$$

where $\Delta=a-2 x u+u^{2}-2 x v+2 u v+v^{2}$.

Remark 6. Setting $a=1$ in results (49) and (53) and using relation (6), we obtain a known results given in [13].

Further properties for the new extended Gegenbauer polynomials of two variables $C_{n}^{\alpha}(x, y ; a, p)$ can be obtained in the form of the following theorems:

Theorem 7. The following Mellin transform representation of the new extended Gegenbauer polynomials $C_{n}^{\alpha}(x, y ; a, p)$ holds true:

$$
\int_{0}^{\infty} C_{n}^{\alpha}(x, y ; a, p) p^{s-1} d p=\frac{\Gamma(s) \Gamma(\alpha+s)}{\Gamma(\alpha)} C_{n}^{\alpha+s}(x, y ; a), \quad \operatorname{Re}(s)>0, \operatorname{Re}(p)>0 .
$$

Proof. Multiplying both sides of Equation (26) by $p^{s-1}$ and integrating with respect to $p$ between the limits 0 to $\infty$, we get

$$
\int_{0}^{\infty} C_{n}^{\alpha}(x, y ; a, p) p^{s-1} d p=\frac{1}{\Gamma(\alpha) n !} \int_{0}^{\infty} t^{\alpha-1} \exp (-a t) H_{n}(2 x t,-y t) \int_{0}^{\infty} \exp \left(-p t^{-1}\right) p^{s-1} d p d t
$$

Now, using the following relation [18]:

$$
\int_{0}^{\infty} \exp \left(-p t^{-1}\right) p^{s-1} d p=t^{s} \Gamma(s)
$$

in the R.H.S. of Equation (55), we get

$$
\int_{0}^{\infty} C_{n}^{\alpha}(x, y ; a, p) p^{s-1} d p=\frac{\Gamma(s)}{\Gamma(\alpha) n !} \int_{0}^{\infty} t^{\alpha+s-1} \exp (-a t) H_{n}(2 x t,-y t) d t,
$$

which on using relation (21) yields assertion (54) of Theorem 7.

For $y=1$ in assertion (54) of Theorem 7 , we get the following result:

Corollary 7. The following Mellin transform representation of the new extended Gegenbauer polynomials $C_{n}^{\alpha}(x ; a, p)$ holds true:

$$
\int_{0}^{\infty} C_{n}^{\alpha}(x ; a, p) p^{s-1} d p=\frac{\Gamma(s) \Gamma(\alpha+s)}{\Gamma(\alpha)} C_{n}^{\alpha+s}(x ; a), \operatorname{Re}(s)>0, \operatorname{Re}(p)>0 .
$$


Theorem 8. The following series representations for the new extended Gegenbauer polynomials $C_{n}^{\alpha}(x, y ; a, p)$ and $C_{2 n}^{\alpha}(x, y ; a, p)$ hold true:

$$
\begin{aligned}
& C_{n}^{\alpha}(x, y ; a, p)=\sum_{k=0}^{\infty} \frac{\Gamma(\alpha-k)(-p)^{k}}{\Gamma(\alpha) k !} C_{n}^{\alpha-k}(x, y ; a), \\
& C_{2 n}^{\alpha}(x, y ; a, p)=\frac{2^{n}(n !)^{2}}{(2 n) ! \Gamma(\alpha)} \sum_{k=0}^{n} \sum_{s=0}^{k} \frac{(-1)^{s}(2 s) ! \Gamma(\alpha+k) y^{k}}{2^{s}(n-k) !(k-s) !(s !)^{2}} C_{2 s}^{\alpha+k}(x, y ; a, p) .
\end{aligned}
$$

Proof of (59). From relation (26), we have

$$
C_{n}^{\alpha}(x, y ; a, p)=\sum_{k=0}^{\infty} \frac{(-p)^{k}}{k !} \frac{1}{\Gamma(\alpha) n !} \int_{0}^{\infty} t^{\alpha-k-1} \exp (-a t) H_{n}(2 x t,-y t) d t
$$

which on using relation (21) yields assertion (59) of Theorem 8.

Proof of (60). Consider the following relation [19]:

$$
H_{2 n}(x, y)=2^{n}(n !)^{2} \sum_{k=0}^{n} \frac{\left[H_{k}(x, y)\right]^{2}}{2^{k}(n-k) !(k !)^{2}} .
$$

Replacing $x$ by $2 x t$ and $y$ by $-y t$ in relation (62) and then multiplying both sides by $t^{\alpha-1} \exp \left(-a t-p t^{-1}\right)$ and integrating the resultant equation with respect to $t$ between the limits 0 to $\infty$, we get

$$
\int_{0}^{\infty} t^{\alpha-1} \exp \left(-a t-p t^{-1}\right) H_{2 n}(2 x t,-y t) d t=\int_{0}^{\infty} t^{\alpha-1} \exp \left(-a t-p t^{-1}\right) 2^{n}(n !)^{2} \sum_{k=0}^{n} \frac{\left[H_{k}(2 x t,-y t)\right]^{2}}{2^{k}(n-k) !(k !)^{2}} d t
$$

Next, using the following relation [19]:

$$
\left[H_{k}(x, y)\right]^{2}=(-2 y)^{k}(k !)^{2} \sum_{s=0}^{k} \frac{(-1)^{s} H_{2 s}(x, y)}{2^{s}(k-s) !(s !)^{2}}
$$

in the right hand side of Equation (63) and interchanging the order of summation and integration, we obtain

$$
\begin{aligned}
& \int_{0}^{\infty} t^{\alpha-1} \exp \left(-a t-p t^{-1}\right) H_{2 n}(2 x t,-y t) d t \\
& =2^{n}(n !)^{2} \sum_{k=0}^{n} \sum_{s=0}^{k} \frac{(-1)^{s} y^{k}}{2^{s}(n-k) !(k-s) !(s !)^{2}} \int_{0}^{\infty} t^{\alpha+k-1} \exp \left(-a t-p t^{-1}\right) H_{2 s}(2 x t,-y t) d t
\end{aligned}
$$

which on using relation (26) yields assertion (60) of Theorem 8 . Thus the proof of Theorem 8 is completed.

For $y=1$ in assertions (59) and (60) of Theorem 8 , we get the following results:

Corollary 8. The following series representations for the new extended Gegenbauer polynomials $C_{n}^{\alpha}(x ; a, p)$ hold true:

$$
\begin{aligned}
& C_{n}^{\alpha}(x ; a, p)=\sum_{k=0}^{\infty} \frac{\Gamma(\alpha-k)(-p)^{k}}{\Gamma(\alpha) k !} C_{n}^{\alpha-k}(x ; a), \\
& C_{2 n}^{\alpha}(x ; a, p)=\frac{2^{n}(n !)^{2}}{(2 n) ! \Gamma(\alpha)} \sum_{k=0}^{n} \sum_{s=0}^{k} \frac{(-1)^{s}(2 s) ! \Gamma(\alpha+k)}{2^{s}(n-k) !(k-s) !(s !)^{2}} C_{2 s}^{\alpha+k}(x ; a, p) .
\end{aligned}
$$

Remark 7. If we take $a=1$ in relations (54),(58), (59), (60) (66) and (30), we obtain a known corresponding results given in [13].

Author Contributions: All authors contributed equally to the writing of this paper. All authors read and approved the final manuscript.

Conflicts of Interest: "The authors declare no conflict of interest." 


\section{References}

[1] Chaudhry, M. A., \& Zubair, S. M. (1994). Generalized incomplete gamma functions with applications. Journal of Computational and Applied Mathematics, 55(1), 99-123.

[2] Chhajed, P., \& Nagarajan, D. (2012). Generalized incomplete Gamma function and its applications. International Journal of Mathematical Analysis, 4(1), 79-87.

[3] Özergin, E., Özarslan, M. A., \& Altin, A. (2011). Extension of gamma, beta and hypergeometric functions. Journal of Computational and Applied Mathematics, 235(16), 4601-4610.

[4] Pucheta, P. I. (2017). An new extended beta function. International Journal of Mathematics and its Applications, 5, $255-260$.

[5] Shadab, M., Jabee, S., \& Choi, J. (2018). An extended beta function and its application. Journal of Mathematical Sciences, 103, 235-251.

[6] Rainville, E. D. (1971). Special Functions. Macmillan, New York.

[7] Dattoli, G., Lorenzutta, S., \& Cesarano, E. (1999). From Hermite to Humbert polynomials. Rendiconti dell'Istituto di Matematica dell'Università di Trieste, XXXV, 37-48

[8] Appell, P., \& De Fériet, J. K. (1926). Fonctions hypergéométriques et hypersphériques: polynomes d'Hermite. Gauthier-Villars.

[9] Agarwal, P., Choi, J., \& Jain, S. (2015). Extended hypergeometric functions of two and three variables. Communications of the Korean Mathematical Society, 30(4), 403-414.

[10] Chaudhry, M. A., Qadir, A., Srivastava, H. M., \& Paris, R. B. (2004). Extended hypergeometric and confluent hypergeometric functions. Applied Mathematics and Computation, 159(2), 589-602.

[11] Özarslan, M. A., \& Yilmaz, B. (2014). The extended Mittag-Leffler function and its properties. Journal of Inequalities and Applications, 85(1), 1-10.

[12] Parmar, R. K., Choi, J., \& Purohit, S. D. (2019). Further generalization of the extended Hurwitz-Lerch Zeta functions. Boletim da Sociedade Paranaense de Matemática, 37(1), 177-190.

[13] Atash, A. A., \& Al-Gonah, A. A. (2020). A new extensions of Gegenbauer polynomials. Electronic Journal of University of Aden for Basic and Applied Sciences, 1(2), 69-77.

[14] Dattoli, G., Chiccoli, C., Lorenzutta, S., Maino, G., \& Torre, A. (1993). Generalized Bessel functions and generalized Hermite polynomials. Journal of Mathematical Analysis and Applications, 178(2), 509-516.

[15] Khan, N. U., Kamarujjama, M., \& Kashmin, T. (2018). Generating function associated with the product of Gegenbauer polynomials. International Journal of Mathematics and its Applications, 6(1-C), 557-561.

[16] Özmen, N. (2018). Gegenbauer Polinomlari Için Bilineer ve Bilateral Dogurucu Fonksiyonlar. Afyon Kocatepe Üniversitesi Fen Ve Mühendislik Bilimleri Dergisi, 18(1), 162-168.

[17] Qureshi, M. I., Yasmeen \& Pathan, M. A. (2002). Linear and bilinear generating functions involving Gould-Hopper polynomials. Mathematical Sciences Research Journal, 6(9), 449-456.

[18] Gradshteyn, I. S., \& Ryzhik, I. M. (1994). Tables of Integrals, Series, and Products. (edited by Alan Jeffrey, University of New Castle upon Tyne, England), Academic Press.

[19] Cesarano, C. (2015). Integral representations and new generating functions of Chebyshev polynomials. Hacettepe Journal of Mathematics and Statistics, 44(3), 535-546. 